\title{
First-passage-time problem for tracers in turbulent flows applied to virus spreading
}

\author{
Akhilesh Kumar Verma $\odot,{ }^{1, *}$ Akshay Bhatnagar $\odot,{ }^{2, \dagger}$ Dhrubaditya Mitra, ${ }^{2, \ddagger}$ and Rahul Pandit ${ }^{1, \S}$ \\ ${ }^{1}$ Centre for Condensed Matter Theory, Department of Physics, Indian Institute of Science, Bangalore 560012, India \\ ${ }^{2}$ NORDITA, KTH Royal Institute of Technology and Stockholm University, Roslagstullsbacken 23, 10691 Stockholm, Sweden
}

(Received 6 January 2020; accepted 30 June 2020; published 11 August 2020)

\begin{abstract}
We study the spreading of viruses, such as SARS-CoV-2, by airborne aerosols, via a first-passage-time problem for Lagrangian tracers that are advected by a turbulent flow: By direct numerical simulations of the three-dimensional (3D) incompressible Navier-Stokes equation, we obtain the time $t_{R}$ at which a tracer, initially at the origin of a sphere of radius $R$, crosses the surface of the sphere for the first time. We obtain the probability distribution function $\mathcal{P}\left(R, t_{R}\right)$ and show that it displays two qualitatively different behaviors: (a) for $R \ll L_{\mathrm{I}}$, $\mathcal{P}\left(R, t_{R}\right)$ has a power-law tail $\sim t_{R}^{-\alpha}$, with the exponent $\alpha=4$ and $L_{\mathrm{I}}$ the integral scale of the turbulent flow; (b) for $L_{\mathrm{I}} \lesssim R$, the tail of $\mathcal{P}\left(R, t_{R}\right)$ decays exponentially. We develop models that allow us to obtain these asymptotic behaviors analytically. We show how to use $\mathcal{P}\left(R, t_{R}\right)$ to develop social-distancing guidelines for the mitigation of the spreading of airborne aerosols with viruses such as SARS-CoV-2.
\end{abstract}

DOI: 10.1103/PhysRevResearch.2.033239

\section{INTRODUCTION}

By 1 June 2020 (14:31 GMT) the COVID-19 coronavirus pandemic had affected 213 countries and territories and 2 international conveyances; the numbers of cases and deaths were, respectively, 6300444 and 374527 [1]. Social distancing has played an important role in mitigation strategies that have been used in several countries to arrest the spread of COVID-19 [2]. To optimize social-distancing guidelines we must ask the following: How far, and how fast, do small respiratory droplets or virus-bearing aerosols spread in turbulent flows? Given the ongoing COVID-19 pandemic, it is important and extremely urgent to have at least a semiquantitative answer to this question. SARS-CoV-2, the virus that causes COVID-19, spreads, principally, in two different ways: (1) First, respiratory droplets, ejected by the sneeze or cough of a patient, fall on nearby surfaces or persons; in this case, approximate estimates of the distance over which droplets are likely to travel are available [3-5]. (2) Second, transmission of this virus can occur because of airborne aerosols, such as (a) a cloud of fine droplets, with diameters smaller than $5 \mu \mathrm{m}$, emitted by an infected person while speaking loudly [6], or (b) the SARS-CoV-2 RNA on fine, suspended particulate matter [7]. These aerosols may remain suspended in the air for a long time. Indeed, they have been reported in two

\footnotetext{
*akhilesh@iisc.ac.in

†akshayphy@gmail.com

\$dhruba.mitra@gmail.com

$\S$ Also at Jawaharlal Nehru Centre For Advanced Scientific Research, Jakkur, Bangalore, India; rahul@iisc.ac.in

Published by the American Physical Society under the terms of the Creative Commons Attribution 4.0 International license. Further distribution of this work must maintain attribution to the author(s) and the published article's title, journal citation, and DOI.
}

hospitals in Wuhan [8], and there is growing evidence that the SARS-CoV-2 virus could also spread via airborne aerosols [6-10], typically indoors [11]. Other diseases can also spread because of airborne aerosols; examples include measles [12], chickenpox [13], tuberculosis [14], and avian flu [15].

The typical sedimentation speed for such aerosols is comparable to their thermal speed. Therefore, at the simplest level, it is natural to model these aerosol particles as neutrally buoyant Lagrangian tracers, which are advected by the flow but are passive, in the sense that they do not affect the flow velocity. We can then study the spread of viruses, such as SARS-CoV-2, via the airborne-aerosol route, by considering the advection of such tracers by turbulent fluid flows. There have been extensive studies of such tracers in the fluiddynamics literature [16,17], and models for such tracers have been used, inter alia, to model the dispersion of pheromones by lepidoptera [18].

We would like to determine the time that an aerosol particle (one of the red particles in the schematic diagram of Fig. 1) takes to travel a distance $R$ from its source (the man at the center of Fig. 1). In a turbulent flow, this time is random; furthermore, a tracer particle can go past the distance $R$, turn back, and reach $R$ again. It is important, therefore, to calculate the time it takes for an aerosol particle to reach the distance $R$ for the first time and to calculate the probability distribution function (PDF) of the first-passage time of a tracer in a turbulent flow. We carry out this calculation below.

Specifically, we consider Lagrangian tracer particles that emanate from a point source in a turbulent fluid. If $t_{R}$ is the time at which a tracer, initially at the origin of a sphere of radius $R$, crosses the surface of the sphere for the first time, what is the probability distribution function (PDF) $\mathcal{P}\left(R, t_{R}\right)$ ? The answer to this question is of central importance in both fundamental nonequilibrium statistical mechanics [19-23] and in understanding the dispersal of tracers by a turbulent flow, a problem whose significance cannot be overemphasized, for it is of relevance to the advection of (a) airborne viruses, as 


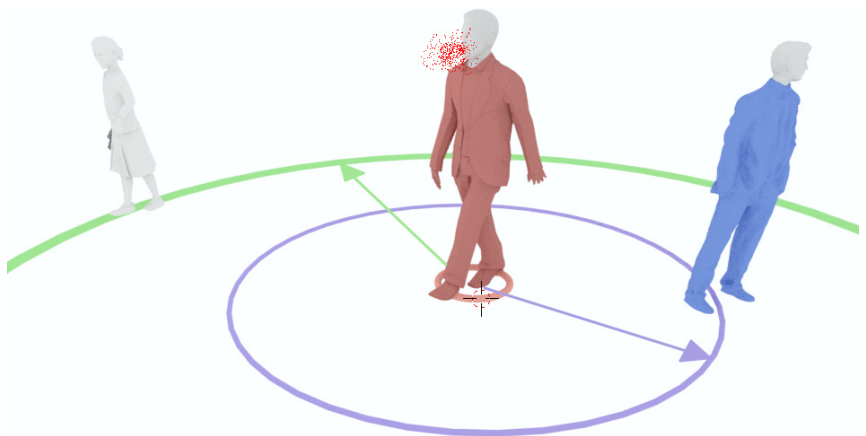

FIG. 1. A schematic diagram illustrating how aerosols with viruses (red points) may be advected by a turbulent flow, from the person at the center (the source) to other persons at different distances from the center.

we have noted above, and (b) pollutants in the atmosphere. First-passage-time problems have been studied extensively [20-23] and they have found applications in a variety of areas in physics and astronomy, chemistry [24], biology [25], and finance [26]. In the fluid-turbulence context, different groups have studied zero crossings of velocity fluctuations [27] or various statistical measures of two-particle dispersion, including exit-time statistics for such dispersion in two- and three-dimensional (2D and 3D) turbulent flows [28,29]. In contrast to these earlier studies (e.g., Refs. [28-30]), the first-passage-time problem we pose considers one tracer in a turbulent flow that is statistically homogeneous and isotropic. For such a particle we show, via extensive direct numerical simulations (DNSs), that $\mathcal{P}\left(R, t_{R}\right)$ displays a crossover between two qualitatively different behaviors: (a) For $R \ll L_{\mathrm{I}}$, $\mathcal{P}\left(R, t_{R}\right) \sim t_{R}^{-\alpha}$, with $L_{\mathrm{I}}$ the integral scale of the turbulent flow and the exponent $\alpha=4$; and (b) for $L_{\mathrm{I}} \lesssim R, \mathcal{P}\left(R, t_{R}\right)$ has an exponentially decaying tail (Fig. 2). We develop models that allow us to obtain these two asymptotic behaviors analytically. Most important, we show how to use $\mathcal{P}\left(R, t_{R}\right)$ to obtain estimates of social-distancing guidelines for the mitigation of the spreading of airborne aerosols with viruses such as SARS-CoV-2.

\section{MODELS, METHODS, AND RESULTS}

The 3D incompressible Navier-Stokes equation is

$$
\partial_{t} \boldsymbol{u}+(\boldsymbol{u} \cdot \nabla) \boldsymbol{u}=-\nabla p+v \nabla^{2} \boldsymbol{u}+\boldsymbol{f},
$$

and

$$
\nabla \cdot \boldsymbol{u}=0 .
$$

Here, $\boldsymbol{u}(\boldsymbol{x}, t)$ is the Eulerian velocity at position $\boldsymbol{x}$ at time $t, p(x, t)$ is the pressure field, and $v$ is the kinematic viscosity of the fluid; the constant density is chosen to be unity. Our direct numerical simulation (DNS) uses the pseudospectral method [31], with the $2 / 3$ rule for dealiasing, in a triply periodic cubical domain with $N^{3}$ collocation points; we employ the second-order exponential Adams-Bashforth scheme for time stepping [32]. We obtain a nonequilibrium statistically stationary turbulent state via a forcing term $\boldsymbol{f}$, which imposes a constant rate of energy injection [33,34], in wave-number shells $k=1$ and $k=2$ in Fourier space; this turbulent state is statistically homogeneous and isotropic.

To obtain the statistical properties of Lagrangian tracers, which are advected by this turbulent flow, we seed the flow with $\mathcal{N}_{\mathrm{p}}$ independent identical tracer particles. If the Lagrangian displacement of a tracer, which was at position $\mathbf{r}_{0}$ at time $t_{0}$, is $\boldsymbol{r}\left(t \mid \mathbf{r}_{0}, t_{0}\right)$, then its temporal evolution is given by

$$
\frac{d}{d t} \boldsymbol{r}=\boldsymbol{v}\left(t \mid \mathbf{r}_{0}, t_{0}\right)=\boldsymbol{u}(\boldsymbol{r}, t),
$$

where $\boldsymbol{v}$ is its Lagrangian velocity. In Eq. (2), we need the Eulerian flow velocity at off-grid points; we obtain this by trilinear interpolation, and we use the first-order Euler method for time marching (see, e.g., Ref. [32]). We give important parameters for our DNS runs in Table I. These include the time step $d t$, the energy dissipation rate $\epsilon=2 v \sum_{k} k^{2} E(k)$, where $E(k)=\sum_{k-1 / 2<k^{\prime}<k+1 / 2} \boldsymbol{u}\left(\mathbf{k}^{\prime}\right) \cdot \boldsymbol{u}\left(-\mathbf{k}^{\prime}\right)$ is the energy
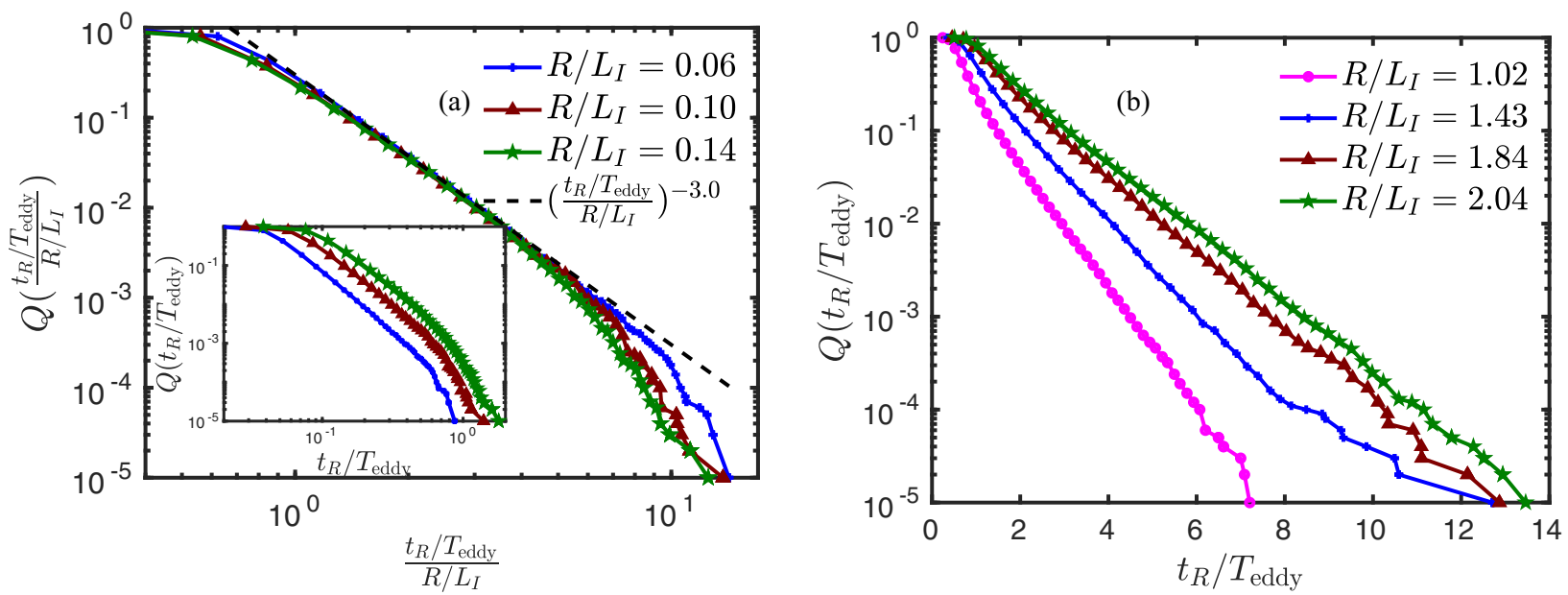

FIG. 2. Plots of the complementary cumulative probability distribution functions (CPDFs) $\mathcal{Q}$ vs the scaled first-passage time $t_{R}$ (see text):

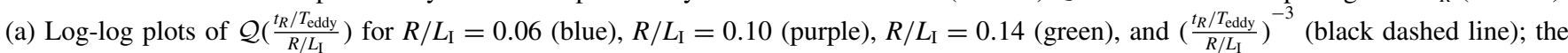
inset shows log-log plots of $\mathcal{Q}\left(t_{R} / T_{\text {eddy }}\right)$ for the same values of $R / L_{\mathrm{I}}$. (b) Semilog plots of $\mathcal{Q}\left(t_{R} / T_{\text {eddy }}\right)$ for $R / L_{\mathrm{I}}=1.02$ (pink), $R / L_{\mathrm{I}}=1.43$ (blue), $R / L_{\mathrm{I}}=1.84$ (purple), and $R / L_{\mathrm{I}}=2.04$ (green). 
TABLE I. Parameters (see text for definitions) for our DNS runs: $N^{3}$ is the total number of collocation points; $v$ is the kinematic viscosity; $d t$ is the time step; $\mathrm{Re}_{\lambda}$ is the Taylor-microscale Reynolds number; $\epsilon$ is the energy dissipation rate; $\eta$ is the Kolmogorov dissipation length scale; $k_{\max }$ is the maximum wave number that we use; $L_{\mathrm{I}}$ is the integral length scale; $T_{\text {eddy }}$ is the integral-scale eddy-turnover time; $\tau_{\eta}=(\nu / \epsilon)^{1 / 2}$ is the Kolmogorov dissipation time scale; and $\mathcal{N}_{\mathrm{p}}$ is the number of tracer particles.

\begin{tabular}{cccccccccccc}
\hline \hline$N$ & $v$ & $d t$ & $\operatorname{Re}_{\lambda}$ & $\epsilon$ & $\eta$ & $k_{\max } \eta$ & $\lambda$ & $L_{\mathrm{I}}$ & $T_{\text {eddy }}$ & $\tau_{\eta}$ & $\mathcal{N}_{\mathrm{p}}$ \\
\hline 512 & $1.2 \times 10^{-3}$ & $2 \times 10^{-4}$ & 82 & 0.67 & $7.12 \times 10^{-3}$ & 1.21 & 0.08 & 0.49 & 0.43 & $4.23 \times 10^{-2}$ & 100000 \\
\hline
\end{tabular}

spectrum, the Taylor-microscale $\lambda=\sqrt{\frac{2 \nu E}{\epsilon}}$, where the total energy $E=\sum_{k} E(k)$, the Taylor-microscale Reynolds number $\operatorname{Re}_{\lambda} \equiv \lambda u_{\mathrm{rms}} / v$, where $u_{\mathrm{rms}}=\sqrt{2 E}$ is the root-meansquare velocity of the flow; $L_{\mathrm{I}}=\frac{\sum_{k} E(k) / k}{\sum_{k} E(k)}$ is the integral length scale and $T_{\text {eddy }}=L_{\mathrm{I}} / u_{\mathrm{rms}}$ is the integral-scale eddyturnover time; $\eta=\left(v^{3} / \epsilon\right)^{1 / 4}$ and $\tau_{\eta}=(v / \epsilon)^{1 / 2}$ are, respectively, the Kolmogorov dissipation length and timescale; and $k_{\max }$ is the maximum wave number that we use in our DNS.

Clearly, $t_{R}$ is the first time at which $|\boldsymbol{r}|$ becomes equal to $R$. Instead of computing the PDF (or histogram) of $t_{R}$ numerically, we calculate the complementary cumulative probability distribution function (CPDF) $\mathcal{Q}\left(t_{R}\right)$ by using the rank-order method [35] to circumvent binning errors. In Fig. 2, we present $\log -\log$ and semilog plots of $\mathcal{Q}\left(t_{R}\right)$ vs $t_{R} / T_{\text {eddy }}$ for several values of $R$. From Fig. 2(a) we conclude that, for $R \ll L_{\mathrm{I}}, \mathcal{Q}\left(t_{R} / T_{\text {eddy }}\right) \sim\left(t_{R} / T_{\text {eddy }}\right)^{-\alpha+1}$, for large $t_{R} / T_{\text {eddy }}$, with $\alpha \simeq 4$; note that, in this power-law scaling regime, the complementary CPDFs for different values of $R / L_{\mathrm{I}}$ collapse onto a universal scaling form, if we plot $\mathcal{Q}\left(\frac{t_{R} / T_{\text {eddy }}}{R / L_{\mathrm{I}}}\right)$. In contrast, Fig. 2(b) shows that, for $L_{\mathrm{I}} \lesssim R$, the tail of $\mathcal{Q}\left(t_{R} / T_{\text {eddy }}\right)$ decays exponentially. For the first-passage-time PDF, these results imply that

$$
\mathcal{P}\left(R, t_{R} / T_{\text {eddy }}\right) \sim \begin{cases}\left(t_{R} / T_{\text {eddy }}\right)^{-4} & \text { for } R \ll L_{\mathrm{I}}, \\ \exp \left[-\left(t_{R} / T_{\text {eddy }}\right)\right] & \text { for } \quad L_{\mathrm{I}} \lesssim R .\end{cases}
$$

We now develop models that allow us to understand these two asymptotic behaviors analytically.

For the power-law behavior of $\mathcal{P}\left(R, t_{R}\right)$, in the range $R \ll$ $L_{\mathrm{I}}$, we construct the following natural ballistic model: Tracer particles emanate from the origin with (a) a velocity whose magnitude $v$ is a random variable with a PDF $p(v)$, and (b) when it starts out from the origin, the tracer's velocity vector points in a random direction. Tracers move ballistically for short times. Therefore, for $R \ll L_{\mathrm{I}}$, the first-passage time $t_{R}=$ $R / v$, and the first-passage PDF is

$$
\mathcal{P}\left(R, t_{R}\right)=\int \delta\left(t_{R}-R / v\right) p(v) d v .
$$

In statistically homogeneous and isotropic and incompressible-fluid turbulence, each component of the Eulerian velocity has a PDF that is very close to Gaussian [36], so $p(v)$ has the Maxwellian [37] form

$$
p(v)=C_{d} v^{d-1} \exp \left(-v^{2} / \sigma^{2}\right),
$$

where $C_{d}$ depends on the spatial dimension $d$, and $C_{d}=4 \pi$ for $d=3$, and $\sigma=\sqrt{\left\langle v^{2}\right\rangle}=u_{\mathrm{rms}}$. We substitute Eq. (5) in
Eq. (4); then, by integrating over $v$, we obtain

$$
\mathcal{P}\left(R, t_{R}\right)=C_{d} \frac{R^{3}}{t_{R}^{d+1}} \exp \left[-R^{2} /\left(t_{R}^{2} \sigma^{2}\right)\right] .
$$

Therefore, in the limit of small $R$ and large $t_{R}$, the firstpassage-time probability is

$$
\mathcal{P}\left(R, t_{R}\right) \sim R^{3} / t_{R}^{4}, \quad \text { for } d=3 ;
$$

this power-law exponent is the same as the one we have obtained from our DNSs above (Table I and Fig. 2).

We can obtain the tail $\mathcal{P}\left(R, t_{R} / T_{\text {eddy }}\right) \sim \exp \left[-\left(t_{R} / T_{\text {eddy }}\right)\right]$ for $L_{\mathrm{I}} \lesssim R$ as follows. At times that are larger than the typical autocorrelation time of velocities in the Lagrangian description, we follow Taylor [38] and assume that the motion of a tracer particle is diffusive. Therefore, we consider a Brownian particle in three dimensions (3D). To calculate the first-passage-time PDF, we must first obtain the survival probability $S(t, R \mid 0)$, i.e., the probability that the particle has not reached the surface of the sphere of radius $R$ up to time $t$, if it has started from the origin of this sphere. We start with the forward Fokker-Planck equation [22,39] for the PDF of finding the particle at a distance $r$ from the origin at time $t$,

$$
\frac{\partial P(r, t)}{\partial t}=K\left(\frac{\partial^{2}}{\partial r^{2}}+\frac{2}{r} \frac{\partial}{\partial r}\right) P(r, t),
$$

where $K$ is the diffusion constant; this PDF satisfies the initial condition $P(r, 0)=\delta(r) /\left(4 \pi r^{2}\right)$ and the absorbing boundary condition $P(R, t)=0$ for all $t$ at $r=R$. We obtain the following solution,

$$
P(r, t)=\frac{1}{2 R^{2}} \sum_{n=0}^{\infty} \frac{n}{r} \sin \left(\frac{n \pi r}{R}\right) \exp \left(-K n^{2} \pi^{2} t / R^{2}\right),
$$

whence we get

$$
\begin{aligned}
S\left(R, t_{R}\right) & =\int_{0}^{R} P(r, t) 4 \pi r^{2} d r \\
& =2 \sum_{n=0}^{\infty}(-1)^{n+1} \exp \left(-K n^{2} \pi^{2} t / R^{2}\right),
\end{aligned}
$$

where, in the last step, we have used Eq. (9). The first-passagetime probability is

$$
\begin{aligned}
\mathcal{P}\left(R, t_{R}\right) & =-\frac{\partial}{\partial t_{R}} S\left(R, t_{R}\right) \\
& =\frac{2 K \pi^{2}}{R^{2}} \sum_{n=0}^{\infty}(-1)^{n+1} n^{2} \exp \left(-K n^{2} \pi^{2} t_{R} / R^{2}\right) .
\end{aligned}
$$



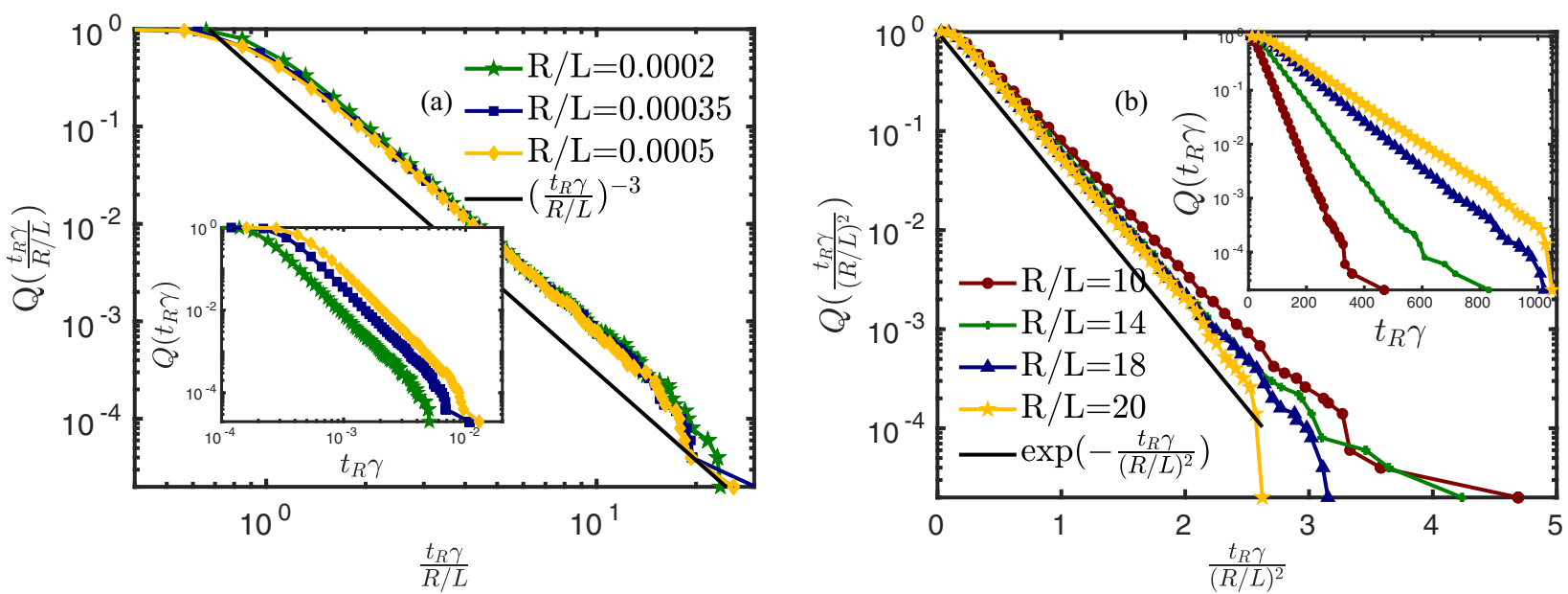

FIG. 3. (a) Log-log plots of the complementary CPDFs $\mathcal{Q}\left(\frac{\gamma t_{R}}{R / L}\right)$ of the scaled first-passage time $\frac{\gamma t_{R}}{R / L}$, for $R \ll L$ and $\gamma=0.01$; the complementary CPDFs, for $R / L=0.0002$ (green), $R / L=0.00035$ (blue), and $R / L=0.0005$ (orange), collapse onto one curve; (b) semilog plots of the complementary CPDFs of the scaled first-passage time $t_{R} / R^{2}$, for $L \lesssim R$ and $\gamma=30$. The complementary CPDF of $t_{R} / R^{2}$, for $R / L=10$ (purple), $R / L=14$ (green), $R / L=18$ (blue), and $R / L=20$ (orange), collapse onto one curve. Plots of the complementary CPDFs $\mathcal{Q}\left(\gamma t_{R}\right)$ vs $\gamma t_{R}$ are shown in the insets.

At large times, the first term $(n=1)$ is the dominant one; therefore,

$$
\mathcal{P}\left(R, t_{R}\right) \sim\left(1 / R^{2}\right) \exp \left(-K \pi^{2} t_{R} / R^{2}\right),
$$

the exponential form that we have obtained from our DNS [Fig. 2(b)]; the $1 / R^{2}$ prefactor cannot be extracted reliably from our DNS data, because this requires much longer runs than are possible with our computational resources.

We now show that both the small- and large- $R / L_{\mathrm{I}}$ behaviors of $\mathcal{P}\left(R, t_{R}\right)\left(R, t_{R}\right)$ in Eq. (3) can be obtained from one stochastic model for the motion of a particle. The simplest such model uses a particle that obeys the following Ornstein-Uhlenbeck (OU) model,

$$
\begin{aligned}
& \frac{d x_{i}}{d t}=v_{i}, \\
& \frac{d v_{i}}{d t}=-\gamma v_{i}+\frac{\sqrt{\Gamma}}{m} \zeta_{i} .
\end{aligned}
$$

Here, $\gamma$ and $\Gamma$ are positive constants; $x_{i}$ and $v_{i}$ are the Cartesian components of the position and velocity of the particle; in three dimensions, $i=1,2$, and $3 ; \zeta_{i}(t)$ is a zero-mean Gaussian white noise with $\left\langle\zeta_{i}\right\rangle=0$ and $\left\langle\zeta_{i}(t) \zeta_{j}\left(t^{\prime}\right)\right\rangle=\delta_{i j} \delta\left(t-t^{\prime}\right)$; this noise is such that the fluctuation-dissipation theorem (FDT) holds. Note that there is no FDT for turbulence. However, for the one-particle statistics that we consider, the simple OU model is adequate. We use $N_{p}=50000$ particles; for each particle, the initial-position components $x_{i}(t=0)$ are distributed randomly and uniformly on the interval $[0,2 \pi]$, and the velocity components $v_{i}(t=0)$ are chosen from a Gaussian distribution. For each particle, we obtain numerically the time $t_{R}$ at which it reaches a distance $R$ from the origin for the first time. We then obtain the first-passage-time complementary CPDF $\mathcal{Q}\left(t_{R}\right)$, which we plot in Fig. 3, for $R \ll L$ and $L \lesssim R$, where $L=\sqrt{\frac{\Gamma}{\gamma^{3}}}$, the natural length scale for Eq. (13), plays the role of $L_{\mathrm{I}}$ in our DNSs above (Table I and Fig. 2). We find

$$
\begin{aligned}
& \mathcal{P}\left(R, t_{R}\right) \sim\left[\frac{\gamma t_{R}}{(R / L)}\right]^{-4}, \quad \text { for } R \ll L, \\
& \mathcal{P}\left(R, t_{R}\right) \sim \exp \left(-\frac{\gamma t_{R}}{(R / L)^{2}}\right), \quad \text { for } L \lesssim R ;
\end{aligned}
$$

these are the OU-model analogs of our DNS results Eq. (3). We have carried out two OU-model simulations: (a) We have designed the first, with $\gamma=0.01$, to explore the form of $\mathcal{P}\left(R, t_{R}\right)$ in the ballistic regime $R \ll L$; (b) the second, with $\gamma=30$, allows us to uncover the form of $\mathcal{P}\left(R, t_{R}\right)$ in the diffusive regime $L \lesssim R$. [From a numerical perspective, it is expensive to obtain the precise form of $\mathcal{P}\left(R, t_{R}\right)$ in both ballistic and diffusive regimes, with one value of $\gamma$.] We now explore in detail the forms of $\mathcal{P}\left(R, t_{R}\right)$ in these two regimes. In Fig. 3(a), we present log-log plots of the complementary CPDFs of the scaled first-passage time $t_{R} / R$, for $R \ll L$ and $\gamma=0.01$. The complementary CPDFs of $t_{R} / R$, for $R / L=0.0002, R / L=0.00035$, and $R / L=0.0005$, collapse onto one curve, i.e., in this regime, $t_{R}$ scales as $R$, which is a clear manifestation of ballistic motion. In Fig. 3(b), we present semilog plots of the complementary CPDFs of the scaled first-passage time $t_{R} / R^{2}$, for $L \lesssim R$ and $\gamma=30$. The complementary CPDFs of $t_{R} / R^{2}$, for $R / L=10, R / L=14$, $R / L=18$, and $R / L=20$, collapse onto one curve; from this we conclude that, in this regime, $t_{R}$ scales as $R^{2}$, which is a clear signature of diffusive motion.

\section{CONCLUSIONS AND DISCUSSION}

We have defined and studied a first-passage-time problem for Lagrangian tracers that are advected by a 3D turbulent flow that is statistically steady, homogeneous, and isotropic. Our work shows that the first-passage-time $\operatorname{PDF} \mathcal{P}\left(R, t_{R}\right)$ has tails that cross over from a power-law form to an exponentially decaying form as we move from the regime $R \ll L_{\mathrm{I}}$ to $L_{\mathrm{I}} \lesssim R$ [Eq. (3)]. We develop ballistic-transport and diffusive models, 

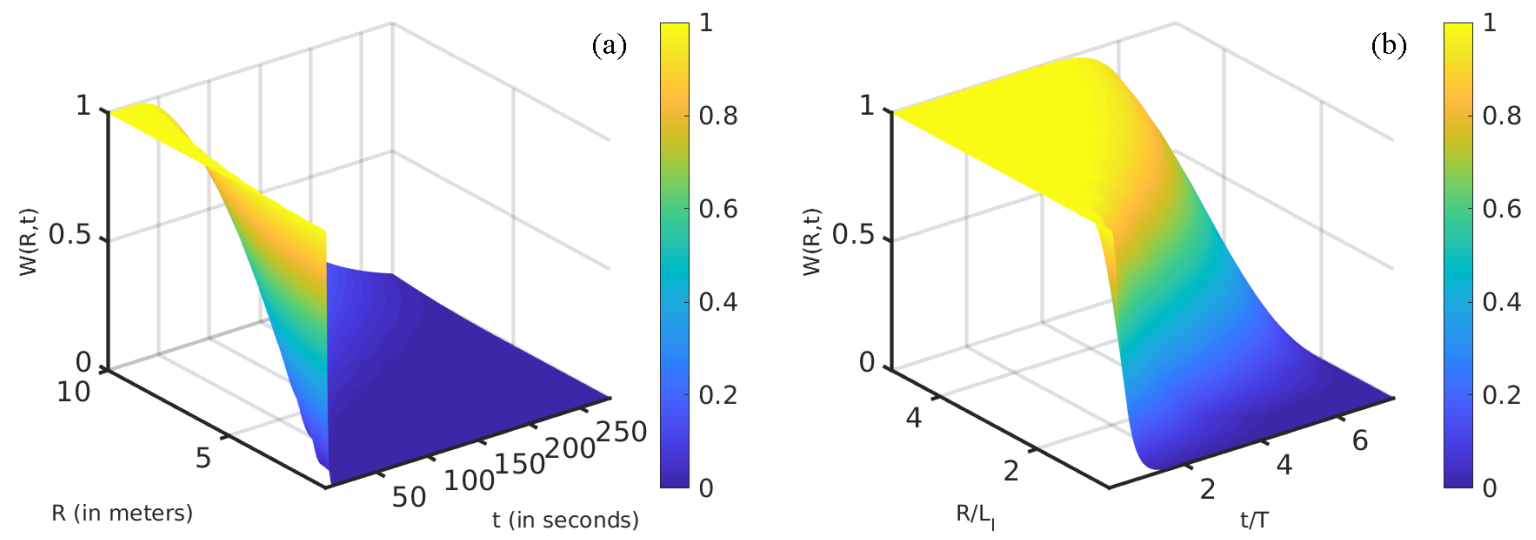

FIG. 4. Surface plots of $W(R, t)$, the probability of virus-laden aerosol particles not reaching a person (at a distance $R$ from an infected person), up until time $t$ (the first-passage time) in (a) vs $R$ and $t$, and (b) vs $R / L_{I}$ and $t / T$, in the diffusive region, for the representative values $K=0.1 \mathrm{~m}^{2} / \mathrm{s}, u_{\mathrm{rms}}=0.05 \mathrm{~m} / \mathrm{s}$, and $L_{\mathrm{I}}=2 \mathrm{~m}$.

for which we can obtain these limiting asymptotic behaviors of $\mathcal{P}\left(R, t_{R}\right)$ analytically. We also demonstrate that an $\mathrm{OU}$ model, with Gaussian white noise, which mimics the effects of turbulence, suffices to obtain the crossover between these limiting forms. Of course, such a simple stochastic model cannot be used for more complicated multifractal properties of turbulent flows $[29,36,40]$.

Earlier studies have concentrated on two-particle relative dispersion by using doubling-time statistics, in 2D fluid turbulence; in particular, they have shown that the PDF of this doubling time has an exponential tail [28]. Studies of velocity zero crossings [27], in a turbulent boundary layer, have shown that PDFs of the zero-crossing times have exponential tails.

The single-particle first-passage-time statistics that we study still needs to be explored. Furthermore, $\mathcal{P}\left(R, t_{R}\right)$ can be used to develop social-distancing guidelines for the mitigation of the spreading of airborne aerosols with viruses such as SARS-CoV-2 as we show below.

Given a pseudospectral DNS of the type we have carried out, we can obtain the integral scale $L_{\mathrm{I}}$ and $u_{\text {rms }}$ from the energy spectrum $E(k)$, as we have noted above. A recent study of COVID-19 in 320 municipalities in China suggests that a very large fraction of COVID-19 infections occur because of indoor transmission of the SARS-CoV-2 virus [11]. Therefore, it is important to study such transmission in rooms and offices; a comprehensive DNS study of the Navier-Stokes equation, with the correct boundary conditions enforced at every wall and surface in the room and accurate forcing functions (dictated, e.g., by fans and vents), is a considerable challenge. Furthermore, it is not possible to carry out such a DNS for every room with a different arrangement of furniture in it. Hence, it is important to come up with semiquantitative criteria that help us to understand and mitigate the indoor transmission of such viruses. Turbulence models have been used to study the flow of air in rooms and offices [41,42]; from these models and related experiments we obtain the estimate $u_{\mathrm{rms}} \simeq 0.05 \mathrm{~m} / \mathrm{s}$ in a typical office. We must also estimate $L_{\mathrm{I}}$, for it is an important crossover length scale in our analysis of $\mathcal{P}\left(R, t_{R}\right)$. In our DNS, $L_{\mathrm{I}}$ is $\simeq 0.1 L$, where $L$ is the linear size of our simulation domain. In a typical office or a train, with fixed forcing, via fans or vents, we use $L_{\mathrm{I}}$ to be approximately a few meters; of course, $L_{\mathrm{I}}$ must depend on the degree of crowding on a train or the number of cubicles in a large office room. Now consider one infected person who is at a distance $R$ from another person. The probability of virus-laden aerosol particles not reaching the second person, up until time $t$, is related to $\mathcal{P}\left(R, t_{R}\right)$ as follows,

$$
W(R, t)=1-\frac{\mathcal{P}\left(R, t_{R}=t\right)}{\Sigma_{t_{R}} \mathcal{P}\left(R, t_{R}\right)},
$$

which we calculate, by using Eq. (11), and depict in Figs. 4(a) and 4(b), in the diffusive regime; Fig. 4(a) is a surface plot of

TABLE II. Table of values of $W(R, t)$, the probability of virus-laden aerosol particles not reaching a person (at a distance $R$ from an infected person), up until time $t$ (the first-passage time) in the diffusive region, for the representative values $K=0.1 \mathrm{~m}^{2} / \mathrm{s}, u_{\mathrm{rms}}=0.05 \mathrm{~m} / \mathrm{s}$, and $L_{\mathrm{I}}=2 \mathrm{~m}$.

\begin{tabular}{|c|c|c|c|c|c|c|c|c|}
\hline & $t=10 \mathrm{~s}$ & $t=30 \mathrm{~s}$ & $t=60 \mathrm{~s}$ & $t=100 \mathrm{~s}$ & $t=120 \mathrm{~s}$ & $t=150 \mathrm{~s}$ & $t=180 \mathrm{~s}$ & $t=240 \mathrm{~s}$ \\
\hline$R=2 \mathrm{~m}$ & 0.169 & 0.001 & 0.000 & 0.000 & 0.000 & 0.000 & 0.000 & 0.000 \\
\hline$R=3 \mathrm{~m}$ & 0.642 & 0.074 & 0.003 & 0.000 & 0.000 & 0.000 & 0.000 & 0.000 \\
\hline$R=4 \mathrm{~m}$ & 0.917 & 0.313 & 0.049 & 0.004 & 0.001 & 0.000 & 0.000 & 0.000 \\
\hline$R=5 \mathrm{~m}$ & 0.989 & 0.594 & 0.187 & 0.039 & 0.018 & 0.005 & 0.001 & 0.000 \\
\hline$R=6 \mathrm{~m}$ & 0.999 & 0.805 & 0.383 & 0.129 & 0.074 & 0.032 & 0.014 & 0.002 \\
\hline$R=7 \mathrm{~m}$ & 1.000 & 0.923 & 0.579 & 0.263 & 0.174 & 0.093 & 0.049 & 0.011 \\
\hline$R=8 \mathrm{~m}$ & 1.000 & 0.097 & 0.739 & 0.412 & 0.299 & 0.182 & 0.107 & 0.030 \\
\hline$R=9 \mathrm{~m}$ & 1.000 & 0.099 & 0.850 & 0.553 & 0.428 & 0.283 & 0.180 & 0.059 \\
\hline$R=10 \mathrm{~m}$ & 1.000 & 0.998 & 0.920 & 0.673 & 0.547 & 0.386 & 0.260 & 0.093 \\
\hline
\end{tabular}


$W(R, t)$ vs $R$ and $t$, for the representative values $L_{\mathrm{I}}=2 \mathrm{~m}$ and $u_{\mathrm{rms}}=0.05 \mathrm{~m} / \mathrm{s}$; Fig. 4(b) gives a surface plot of $W$ versus the dimensionless parameters $R / L_{\mathrm{I}}$ and $t / T$, where $T=L_{\mathrm{I}} / u_{\mathrm{rms}}$. (We give similar plots for the ballistic regime in the Supplemental Material [43].) In Table II we give the values of $W(R, t)$ for different values of $R$ and $t$. These figures and Table II lead to three clear observations:

(1) If the separation $R \ll L_{\mathrm{I}}$, i.e., we have to consider the ballistic regime (see the Supplemental Material [43]), then $W(R, t)$ goes very rapidly to 0 (i.e., the aerosol particle reaches the second person), even if $t$ is very small.

(2) The smaller the separation $R$ between two persons, the shorter the time $t$ in which $W(R, t)$ becomes very small, i.e., the aerosol particles reach from one person to the other.

(3) Our calculation leads to quantitative predictions, e.g., if the separation $L_{\mathrm{I}} \lesssim R$, i.e., we have to consider the diffusive regime, then $W(R, t)$ goes to 0 in tens of seconds, if $R=2 \mathrm{~m}$, and in hundreds of seconds, if $R-10 \mathrm{~m}$, for the representative parameters that we use to obtain Table II. A recent study [44] has suggested that the SARS-CoV-2 virus remains viable in aerosols for nearly $3 \mathrm{~h}$. Therefore, if the concentration of virus-laden aerosols is high in a poorly ventilated room, then we must employ more stringent socialdistancing norms than are in place now, even if people spend only tens of minutes together in such a room. The methods that we have developed can be applied, mutatis mutandis, (a) in sophisticated models for virus particles or droplets, e.g., those that use inertial particles $[45,46]$ or multiphase flows $[47,48]$, and (b) in turbulent flows that are not statistically homogeneous and isotropic. We will examine these in future work. At the moment, during this Covid-19 pandemic, it is important to use our minimal model to obtain semiquantitative criteria for social-distancing guidelines, as we have done above.

\section{ACKNOWLEDGMENTS}

D.M. and A.B. thank John Wettlaufer for useful discussions. A.K.V. and R.P. thank Jaya Kumar Alageshan for discussions and for help with Fig. 1, and CSIR (IN) and DST (IN) for financial support. A.B. and D.M. are supported by the grant Bottlenecks for Particle Growth in Turbulent Aerosols from the Knut and Alice Wallenberg Foundation (Dnr. KAW 2014.0048); the computations were performed on resources provided by the Swedish National Infrastructure for Computing (SNIC) at PDC and SERC (at IISc). D.M. is supported by two Swedish Research Council Grants No. 638-2013-9243 and No. 2016-05225.
[1] These data are updated in real time at https://www. worldometers.info/coronavirus/.

[2] See, e.g., https://www.cdc.gov/coronavirus/2019-ncov/preventgetting-sick/social-distancing.html.

[3] https://www.nytimes.com/interactive/2020/04/14/ science/coronavirus-transmission-cough-6-feet-ar-ul.html? campaign_id=57\&emc=edit_ne_20200414\&instance_id= $17644 \&$ nl=evening-briefing\&regi_id=75908386\&segment_id $=25175 \&$ te $=1 \&$ user_id $=$ fb4f001a71c3b41abab178e84896d 909 and references therein.

[4] L. Bourouiba, E. Dehandschoewercker, and J. W. M. Bush, Violent respiratory events: On coughing and sneezing, J. Fluid. Mech. 745, 537 (2014).

[5] L. Bourouiba, Turbulent gas clouds and respiratory pathogen emissions: Potential implications for reducing transmission of COVID-19, JAMA, J. Am. Med. Assoc. 323, 1837 (2020).

[6] https://www.electrooptics.com/news/laserimaging-tech-showshow-covid-19-spread-talking.

[7] L. Setti, F. Passarini, G. De Gennaro, P. Baribieri, M. G. Perrone, M. Borelli, J. Palmisani, A. Di Gilio, V. Torboli, A. Pallavicini et al., SARS-Cov-2 RNA found on particulate matter of Bergamo in Northern Italy: First preliminary evidence, Environ. Res. 188, 109754 (2020).

[8] Y. Liu, Z. Ning, Y. Chen et al., Aerodynamic analysis of SARS-CoV-2 in two Wuhan hospitals, Nature (London) 582, 557 (2020).

[9] K. A. Prather, C. C. Wang, and R. T. Schooley, Reducing transmission of SARS-CoV-2, Science 368, 1422 (2020).

[10] G. A. Somsen, C. van Rijn, S. Kooij, R. A. Bem, D. Bonn, Small droplet aerosols in poorly ventilated spaces and SARS-CoV-2 transmission, Lancet Respir. Med. 8, 658 (2020).

[11] H. Qian, T. Miao, L. Liu, X. Zheng, D. Luo, and Y. Li, Indoor transmission of SARS-CoV-2, medRxiv (2020), doi: 10.1101/2020.04.04.20053058.
[12] E. C. Riley, G. Murphy, and R. L. Riley, Airborne spread of measles in a suburban elementary school, Am. J. Epidemiol. 107, 421 (1978).

[13] J. M. Leclair, J. A. Zaia, M. J. Levin, R. G. Congdon, and D. A. Goldmann, Airborne transmission of chickenpox in a hospital, N. Engl. J. Med. 302, 450 (1980).

[14] A. R. Escombe et al., The detection of airborne transmission of tuberculosis from HIV-infected patients, using an in vivo air sampling model, Clin. Infect. Dis. 44, 1349 (2007).

[15] Y. Zhao, B. Richardson, E. Takle, L. Cahi, D. Schmitt, and H. Xin, Airborne transmission may have played a role in the spread of 2015 highly pathogenic avian influenza outbreaks in the United States, Sci. Rep. 9, 11755 (2019).

[16] G. Falkovich, K. Gawędzki, and M. Vergassola, Particles and fields in fluid turbulence, Rev. Mod. Phys. 73, 913 (2001).

[17] F. Toschi, and E. Bodenschatz, Lagrangian properties of particles in turbulence, Annu. Rev. Fluid Mech. 41, 375 (2009).

[18] A. Celani, E. Villermaux, and M. Vergassola, Odor Landscapes in Turbulent Environments, Phys. Rev. X 4, 041015 (2014).

[19] A. J. Bray, S. Majumdar, and G. Scher, Persistence and firstpassage properties in nonequilibrium systems, Adv. Phys. 62, 225 (2013).

[20] S. Chandrasekhar, Stochastic problems in physics and astronomy, Rev. Mod. Phys. 15, 1 (1943).

[21] S. Redner, A Guide to First-Passage Processes (Cambridge University Press, Cambridge, UK, 2001).

[22] V. Balakrishnan, Elements of Nonequilibrium Statistical Mechanics (Ane Books, New Delhi, 2008).

[23] First-Passage Phenomena and Their Applications, edited by S. Redner, R. Metzler, and G. Oshanin (World Scientific, Singapore, 2014).

[24] G. H. Weiss, First passage time problems in chemical physics, Adv. Chem. Phys. 13, 1 (1967). 
[25] L. Ricciardi, A. Crescenzo, V. Giorno, and A. Nobile, An outline of theoretical and algorithmic approaches to first passage time problems with applications to biological modeling, Math. Jpn. 50, 247 (1999).

[26] R. Chicheportiche and J.-P. Bouchaud, in First-Passage Phenomena and Their Applications (Ref. [23]), p. 447.

[27] P. Kailasnath and K. Sreenivasan, Zero crossings of velocity fluctuations in turbulent boundary layers, Phys. Fluids A 5, 2879 (1993).

[28] G. Boffetta and I. M. Sokolov, Statistics of two-particle dispersion in two-dimensional turbulence, Phys. Fluids 14, 3224 (2002).

[29] A. Vulpiani, L. Biferale, G. Boffetta, A. Celani, M. Cencini, and D. Vergni, in Intermittency in Turbulent Flows, edited by J. C. Vassilicos (Cambridge University Press, Cambridge, UK, 2001), p. 223.

[30] C. C. Lalescu and M. Wilczek, How tracer particles sample the complexity of turbulence, New J. Phys. 20, 013001 (2018).

[31] C. Canuto, M. Hussaini, A. Quarteroni, and T. Zang, Spectral Methods in Fluid Dynamics (Springer, Berlin, 1988).

[32] A. Bhatnagar, A. Gupta, D. Mitra, R. Pandit, and P. Perlekar, How long do particles spend in vortical regions in turbulent flows?, Phys. Rev. E 94, 053119 (2016).

[33] A. Lamorgese, D. Caughey, and S. Pope, Direct numerical simulation of homogeneous turbulence with hyperviscosity, Phys. Fluids 17, 015106 (2005).

[34] G. Sahoo, P. Perlekar, and R. Pandit, Systematics of the magnetic-Prandtl-number dependence of homogeneous, isotropic magnetohydrodynamic turbulence, New J. Phys. 13, 013036 (2011).

[35] D. Mitra, J. Bec, R. Pandit, and U. Frisch, Is Multiscaling an Artifact in the Stochastically Forced Burgers Equation? Phys. Rev. Lett 94, 194501 (2005).

[36] R. Pandit, P. Perlekar, and S. S. Ray, Statistical properties of turbulence: An overview, Pramana 73, 157 (2009).

[37] T. Gotoh, D. Fukayama, and T. Nakano, Velocity field statistics in homogeneous steady turbulence obtained using a highresolution direct numerical simulation, Phys. Fluids 14, 1065 (2002).
[38] G. I. Taylor, Diffusion by continuous movements, Proc. London Math. Soc. s2-20, 196 (1922).

[39] H. Z. Risken, The Fokker-Planck Equation (Springer, Berlin, 1989).

[40] A. Arneodo, R. Benzi, J. Berg, L. Biferale, E. Bodenschatz, A Busse, E. Calzavarini, B. Castaing, M. Cencini, L. Chevillard, R. T. Fisher, R. Grauer, H. Homann, D. Lamb, A. S. Lanotte, E. Leveque, B. Luthi, J. Mann, N. Mordant, W.-C. Müller, S. Ott, N. T. Ouellette, J.-F. Pinton, S. B. Pope, S. G. Roux, F. Toschi, H. Xu, and P. K. Yeung, (International Collaboration for Turbulence Research), Universal Intermittent Properties of Particle Trajectories in Highly Turbulent Flows, Phys. Rev. Lett. 100, 254504 (2008).

[41] Y. Li, S. Duan, I. T. Yu, and T. W. Wong, Multi-zone modeling of probable SARS virus transmission by airflow between flats in Block E, Amoy Gardens, Indoor Air 15, 96 (2005).

[42] Z. Zhang, W. Zhang, Z. J. Zhai, and Q. Y. Chen, Evaluation of Various Turbulence Models in Predicting Airflow and Turbulence in Enclosed Environments by CFD: Part 2-Comparison with Experimental Data from Literature, HVACR Res. 13, 871 (2007).

[43] See Supplemental Material at http://link.aps.org/supplemental/ 10.1103/PhysRevResearch.2.033239 for additional figures from our simulation studies.

[44] N. van Doremalen, T. Bushmaker, D. H. Morris et al., Aerosol and surface stability of SARS-CoV-2 as compared with SARSCoV-1, N. Engl. J. Med. 382, 1564 (2020).

[45] M. Cencini, J. Bec, L. Biferale, G. Boffetta, A. Celani, A. Lanotte, S. Musacchio, and F. Toschi, Dynamics and statistics of heavy particles in turbulent flows, J. Turbul. 7, N36 (2006).

[46] J. P. L. C. Salazar, and L. R. Collins, Two-particle dispersion in isotropic turbulent flows, Annu. Rev. Fluid Mech. 41, 405 (2009).

[47] S. Balachandar, and J. K. Eaton, Turbulent dispersed multiphase flow, Annu. Rev. Fluid Mech. 42, 111 (2010).

[48] N. Pal, P. Perlekar, A. Gupta, and R. Pandit, Binary-fluid turbulence: Signatures of multifractal droplet dynamics and dissipation reduction, Phys. Rev. E 93, 063115 (2016). 